\title{
Laser beam makes flies flirt
}

Mind-altering device activates heat-sensitive neural pathways involved in courtship.

\section{Sara Reardon}

28 February 2014

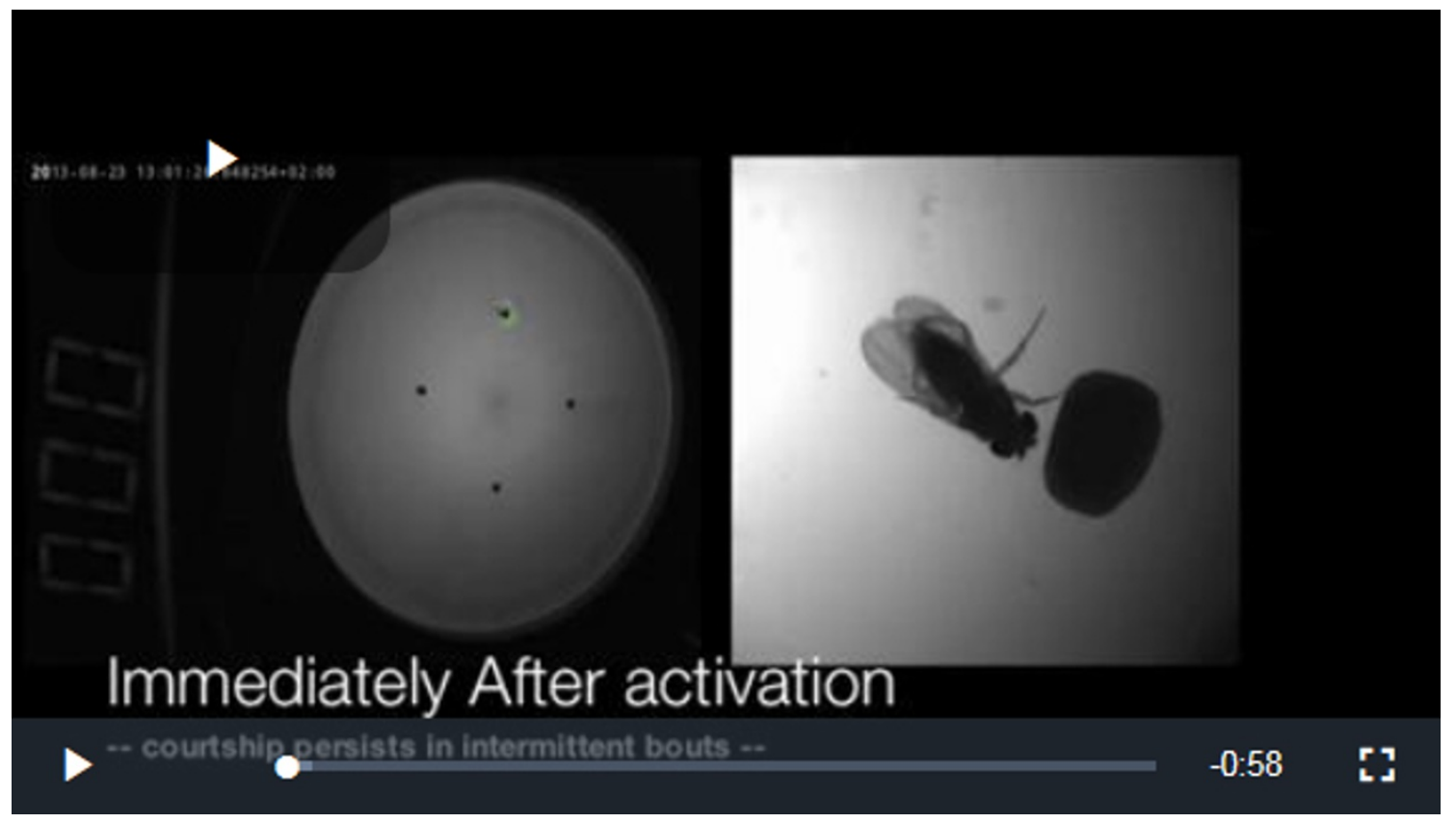

A flipped mental switch is all it takes to make a fly fall in love - even if its object of desire is a ball of wax. A technique called thermogenetics allows researchers to control fly behaviour by activating specific neurons with heat. Combining the system with techniques that use light to trigger neurons could help to elucidate how different neural circuits work together to control complex behaviours such as courtship.

Optogenetics - triggering neurons with light — has been successful in mice but has not been pursued much in flies, says Barry Dickson, a neuroscientist at the Howard Hughes Medical Institute's Janelia Farm Research Campus in Ashburn, Virginia. A fibre-optic cable embedded in a mouse's brain can deliver light to cells genetically engineered to make light-activated proteins, but flies are too small for these fibre optics. Neither will these cells be activated when the flies are put into an illuminated box, because most wavelengths of visible light cannot penetrate a fly's exoskeleton.

Heat can penetrate the exoskeleton, however. Researchers have already studied fly behaviour by adding a heat-activated protein called TRPA1 to neural circuits that control behaviours such as mating and decision-making. When these flies are placed in a hot box, the TRPA1 neurons begin to fire within minutes and drive the fly's actions ${ }^{1}$.

\section{Time flies}

But it would be better to trigger the behaviours more quickly. So Dickson's lab has developed a system called the Fly Mind-Altering Device (FlyMAD), which uses a video camera to track the fly as it moves around in a box. The device then shines an infrared laser at the fly to deliver heat directly to the head. Dickson's group presented the system last October at the Neurobiology of Drosophila conference at Cold Spring Harbor Laboratory in New York, and he is now submitting the work to a peer-reviewed journal.

As proof that the FlyMAD works, the group made flies with TRPA1 in a neural circuit involved in courtship. When the researchers activated the TRPA1 neurons with the laser, the fly began trying to mate with a ball of wax, circling it and 'singing' by vibrating its wings (see 'Laser love'). The fly continued courting for about fifteen minutes after the laser was shut off, suggesting that the heat had 
triggered a lasting, complex behavioural state. The researchers also made flies with TRPA1 in neurons involved in muscular coordination. Switching the laser on instantly made the flies walk backwards. They immediately stopped when it was switched off.

The TRPA1 technique is easier to use than current optogenetics techniques for flies, says Gero Miesenböck of the University of Oxford, UK. His group injected flies' brains with a light-activated chemical that binds to receptors on neurons that make dopamine which causes fear in flies. His group then illuminated the flies with very strong lasers whenever the insect passed through a stream of a harmless gas ${ }^{2}$. This made the fly form false memories and fear the gas. Unlike TRPA1, however, the chemical system could not be reversed when the light was turned off.

Benjamin White, a neuroscientist at the National Institute of Mental Health in Bethesda, Maryland, says that the fine time control could allow fly researchers to start asking the questions that have long been the province of mouse researchers. His group studies how flies make decisions such as where to land or lay eggs, so a system such as FlyMAD could help to decode these snap judgements.

\section{Light work}

Neuroscientist David Anderson of the California Institute of Technology in Pasadena says that TRPA1 switch is exciting because it can be combined with others to determine how different neural circuits work together. He and his colleagues use a different switch: a lightactivated protein called a channelrhodopsin that can respond to long wavelengths of light that can pass through fly exoskeletons. They have used red light to illuminate a box containing flies with this protein, which triggered courtship neurons and caused the male flies to begin singing ${ }^{3}$.

Anderson and Dickson say that they would like to experiment with using both the red and infrared wavelengths to activate circuits right after one another, for instance, to see which circuit controls the other. Lasers could also allow researchers to control two flies while they interact, or to activate mutually exclusive behaviours, such running backwards and forwards at the same time. That way, Anderson says, they can "see which one wins".

Nature I doi:10.1038/nature.2014.14794

\section{References}

1. Pan, Y., Robinett, C. C. \& Baker, B. S. PLoS ONE http://dx.doi.org/10.1371/journal.pone.0021144 (2011).

2. Claridge-Chang, A. et al. Cell 139, 402-415 (2009).

3. Inagaki, H. K. et al. Nature Meth. 11, 325-332 (2014). 